\title{
Stress Response Monitoring of Photoautotrophic Higher Plant Suspension Cultures by Fluorescence Imaging for High-Throughput Toxic Compound Screening
}

\author{
Anna Segečová1,2,3*, Jan Červený1, Thomas Roitsch ${ }^{1,3}$ \\ ${ }^{1}$ Department of Adaptive Biotechnologies, Global Change Research Institute, CAS, Drásov, Czech Republic \\ ${ }^{2}$ Research Centre for Toxic Compounds in the Environment (RECETOX), Masaryk University, Brno, Czech Republic \\ ${ }^{3}$ Departmemt for Plant and Environmental Sciences, Copenhagen Plant Science Centre, University of Copenhagen, Taastrup, \\ Denmark \\ Email: *segecova.a@czechglobe.cz
}

How to cite this paper: Segečová, A., Červený, J. and Roitsch, T. (2017) Stress Response Monitoring of Photoautotrophic Higher Plant Suspension Cultures by Fluorescence Imaging for High-Throughput Toxic Compound Screening. Journal of Environmental Protection, 8, 678-692.

https://doi.org/10.4236/jep.2017.86044

Received: May 9, 2017

Accepted: June 24, 2017

Published: June 27, 2017

Copyright $\odot 2017$ by authors and Scientific Research Publishing Inc. This work is licensed under the Creative Commons Attribution International License (CC BY 4.0).

http://creativecommons.org/licenses/by/4.0/

(c) $\underset{\mathrm{EY}}{\text { (i) Open Access }}$

\begin{abstract}
Photoautotrophic suspension cultures have been established from various model and crop plants and proved to be valuable and robust experimental system to assess coordinated responses of primary and secondary metabolism to metabolic and stress related signals. The use of suspension cultures combines the ease of handling microalgae in microtiter plates with the advantage of testing physiological responses of higher plants, notably in combination with the assessment of the response of photosynthetic activity by PAM chlorophyll fluorescence imaging as well as monitoring changes in secondary metabolite production and ROS formation by steady state fluorescence of plant fluorophores or introduced fluorescent probes. Photoautotrophic cultures provide various advantages as fast, highly sensitive, robust and high-throughput experimental system for screening and characterization of the impact of toxic compounds on higher plants. This opinion article discusses and critically evaluates the potential of photoautotrophic cultures of higher plants in combination with fluorescence imaging assays in microtiter plates as a complement to existing guidelines for testing the toxicity of chemicals in plants.
\end{abstract}

\section{Keywords}

Toxins, Toxicants, Ecotoxicology, PAM Chlorophyll Fluorescence

\section{Introduction}

The very limited number of higher plant test systems currently used for toxic 
compound screening and characterization suffer from various inherent limitations. Toxicity tests on plants are mostly based on growth inhibition and routine assay parameters such as seed germination, root or shoot elongation, wet or dry weight, leaf or frond number are determined [1] [2] [3]. These tests are standardized with ecologically relevant endpoints. However, currently used assays are time and space demanding and therefore unsuited to a rapid screening of toxicants and/or providing insight into the underlying physiological processes.

As an alternative to the plant assays, surrogate toxicity tests on microalgae species are often conducted in microplates as the lifecycle of microalgae compared to higher plants is shorter. Hence, the duration of the test can be reduced by almost an order of magnitude, from 21 days to only 72 to 96 hours [2] [4]. However, these tests involve the inherent uncertainty and thus major drawback of the limited results extrapolation since sensitivity to different toxicants was proven to be highly compound and species specific (reviewed in [5]). Thus, the extrapolation of results from algal toxicity tests to higher plant responses is limited with a certain degree of uncertainty to the general determination of a negative effect of toxicants to photosynthetic organisms, but is not a surrogate for specific plant species of interest.

Additional development and implementation of physiological, biochemical, and molecular endpoints to evaluate phytotoxicity are of interest [5] [6]. For example, [7] concludes that one bioassay parameter cannot generate sufficient information to evaluate comparative phytotoxicity based on shoot and root length, chlorophyll content and root hair damage. Therefore, there is a need to apply several comparative methods based on general stress bioindicators and to incorporate them into a broader series of tests for toxicity. An alternative solution to the slow growth inhibition tests, that does not give any insight into the targets, is method that directly or indirectly determines physiological parameters such as chlorophyll fluorescence (ChlF), multicolor fluorescence (MCF), reactive oxygen species (ROS) probes; oxygen evolution, carbon dioxide assimilation, respiration or transpiration. These methods are well established in ecophysiology or cell physiology. When used in combination with higher plants, however, these methods are time consuming, greenhouse space and labor demanding for testing and routine maintenance, and introduce uncertainty into the results due to the variability among individual plants and/or plant organs. Given these limitations, there is a need for more sensitive, faster and high-throughput screening systems.

\section{Photoautotrophic Higher Plant Suspension Cultures Are Underexplored in Toxicological Research}

Plant cell suspension cultures are individual cells of higher plants cultivated in axenic conditions in liquid medium. Plant cell suspension cultures provide simple model systems for basic plant science research such as the homogeneity of the material, direct and fast accessibility of each single cell to exogenous stimuli as well as easy access of the measured response without interfering cell layers 
with diverse functions or shading, instant availability of biomass depending on the desired cell cycle phase or age of the culture [8] [9]. These features circumvent the challenges associated with the analyses of a multi-factorial plant composed of multiple tissues and cell types exposed to diverse endogenous signals and external factors and/or stimuli. Plant suspension cultures are also valuable in the study of the effects of chemicals and environmental stresses, defense response, secondary metabolite formation, ion transport, gene regulation, and signal transduction, biopharmaceutical production, uptake, metabolism, phytoremediation of toxic compounds and plant resistance to herbicides [9]-[15]. However, so far, only few toxicity tests have been performed with higher plants suspension cultures whereas only endpoints such as biomass after incubation times of more than one week have been determined [16] [17] [18].

While carbon autotrophy is a key property of intact higher plants, tissues or cells rapidly lose their photosynthetic ability, partially due to the effect of the sugars and plant hormones present in the culture medium [19]. Therefore, the photosynthetic activity of suspension cultures is typically low and/or photosynthetically inactive. Photomixotrophic cultures are available from some species which are photosynthetically active but require the presence of exogenous sugars in the culture medium. Photoautotrophic (PA) cultures are available from various dicotyledonous and very few monocotyledonous species, ranging from weed and model species to crop species which grow in the absence of any reduced carbon source [11] [19] [20] [21]. Such cultures combine the advantages of plant suspension cultures with carbon autotrophy.

PA contain chloroplasts and thus anatomically and metabolically correspond to leaf mesophyll cells although differences exist (e.g., in chloroplast number and photosystem activity) [9]. PA cultures were shown to be a suitable model system for mimicking mesophyll cells and for studying the high light intensity acclimation process [8]. The complexity and heterogeneity of the whole plant is thus in PA culture reduced to a population of single mesophyll-like cells or cell clusters. This miniaturization of plant experimental material down to individual cells of PA culture allows to use the high-throughput approach as these suspension cultures can be handled in a similar way as microalgae (e.g., in microtiter plate assays) while retaining the features of a higher plant experimental system [17].

\section{Photoautotrophic Response Monitoring via Fluorescence Imaging}

Stress responses of PA cultures reflect physiological reactions of plant primary and secondary metabolism that can be highly sensitively monitored by fluorescence imaging. Plant suspension cultures are used to assess the impact of various external stimuli on plant cells. These cultures provide simple model systems for plant science research as a uniform cell type is grown under controlled conditions. Contrary to whole plants or plant organs, each single cell in plant suspension culture is exposed to a particular stimulus within seconds [10]. As a result, plant suspension cultures are used to assess fast and transient responses to vari- 
ous stimuli, e.g., to assess defense responses upon stimuli that mimic a pathogen infection via application of fungal elicitors [22] [23]. Even very weak or transient responses such as the activation of signaling components including the activation of MAP kinases, the release of second messengers or formation of ROS can be detected [24]. The introduction of the PA cultures for such studies made it possible to include the metabolic regulation by sugars and to also integrate the regulation of source-sink transitions, and photosynthesis studies including the impact of herbicides [10]. Notably, using PA suspension cultures a coordinated regulation of source-sink transition in combination with the activation of defense responses has been established [24]. The deduced regulatory mechanism that coordinates the induction of sink metabolism and defense responses with a repression of photosynthesis can be verified in various plant pathogen interactions supporting the use of PA cultures [25]. Since the rapid repression of photosynthetic activity in PA cultures by stress related stimuli is a general response, it can also provide a suitable parameter to assess the impact of toxicants.

Photosynthesis is a key process of plant metabolism and is regulated and influenced by the energetic demand of the organism. Photosynthetic processes are also sensitive to damage to photosystems from biotic or abiotic factors, changes in environmental conditions including temperature, $\mathrm{CO}_{2}$ concentration, water availability, light quality and quantity [26] [27]. Changes in photosynthetic activity therefore provide a useful stress biomarker which can be studied by chlorophyll fluorescence [28]. Chlorophyll fluorescence is a result of excess incident photon excitation energy dissipation in photosystems and is one of three possible processes of photon energy conversion along with photochemistry and heat dissipation. Chlorophyll fluorescence can therefore provide an indirect measurement of photosynthetic activity [29].

Pulse amplitude modulated (PAM) fluorometry measures ChlF induction kinetics. This method is based on excitation of dark-adapted photosynthetic material (e.g., leaf or microalgal culture) with various light sources such as weak measuring light (detects fluorescence yield changes), actinic light (drives photosynthetic electron transport) and strong saturating pulses (saturates the capacity of PSII). This technique results in a characteristic fluorescence kinetic from which various ChlF parameters can be derived to analyze the functionality of the photosynthetic apparatus and underlying photochemical and non-photochemical processes [28] [30]. A great advantage of this method is its noninvasiveness.

Another advantage is the introduction of imaging approach into PAM fluorometry allowing the quantitative "machine vision" with spatial resolution. The imaging approach differs from the conventional point fluorometry in that the fluorescence signal is captured from the measured area with charge coupled device (CCD) (e.g., camera). As a result, spatial reconstruction of the measured data for each pixel of the detector is possible, which allows visualization and quantification of the spatial heterogeneity of the photosynthetic activity [30]. Moreover, fluorescence imaging can be used to estimate leaf area for species with relevant leaf architecture [31]. Another advantage of imaging fluorescence 
techniques is very convenient high-throughput data acquisition of many samples at the same time (several plants, leaves or samples in microplates, depending on the detection area of the measuring device) i.e. avoiding any delay between measurements of individual samples. This is in contrast with the common fluorescence microplate readers or non-imaging PAM fluorometers providing only point measurements. Imaging PAM fluorometer measures all samples in the focus area at one time point as a snapshot. Thus the introduction of PAM chlorophyll fluorescence imaging was crucial for precise and non-biased measurement of parallel samples expressing effects with rapid evolution [32]. This physiological high-throughput approach based on PAM ChlF imaging directly assessing photosystem II activity provides the mean for detecting and studying biotic and abiotic stresses, toxicity testing, phenotyping in horticulture and agriculture for identification of tolerant varieties and targeted breeding for improved plant performance [33] [34] [35] [36].

However, as noted in [27] the most useful diagnostic information is obtained when several measured parameters representing a range of plant physiological responses, are combined creating a multi-sensor stress-identification technique. Therefore, combining ChlF with other methods based on different stress markers will eventually result in the development of signatures specific to types of stressors and/or toxicants.

A possible extension of ChlF imaging to monitor the toxicity responses is the application of MCF. MCF is based on the steady state auto-fluorescence of various compounds of plant cells. As described in [37], plant material is excited by long wave UV light which gives rise to a continuous fluorescence emission with four characteristic peaks near to $440 \mathrm{~nm}$ (blue), $520 \mathrm{~nm}$ (green), $690 \mathrm{~nm}$ (red) and $740 \mathrm{~nm}$ (far-red). The blue-green fluorescence is emitted primarily by phenolic compounds and other secondary metabolites, whereas the red and far-red fluorescence comes from chlorophyll. Changes in ratios calculated from these characteristic bands are sensitive to short-term stress (blue/red, blue/far-red) as well as to long term stress conditions (blue/green) [37] [38]. Various types of stress including biotic (pathogen infection) and abiotic (light quality and quantity, water deficiency, pollution and toxicity, nutrients deficiency) were successfully detected by changes in MCF ratios [35] [37] [39] [40]. The use of MCF ratios in turn allows the compilation of a plant stress-catalogue based on MCF signatures [27]. Therefore, we suggest adding MCF into our proposed experimental platform for high-throughput screening of toxic compounds based on fluorescence imaging and PA cell cultures as additional biomarker to ChlF.

Another approach to stress detection based on fluorescence imaging is the determination of ROS production using fluorescent ROS probes. ROS such as singlet oxygen, superoxide and hydrogen peroxide are produced in different cellular compartments including chloroplasts, mitochondria, peroxisomes, plasma membrane and nuclei [41]. Changes in ROS are recognized as regulators of plant responses ranging from the biotic and abiotic stresses to the regulation of normal development [42]. ROS also play a role of oxidants of target mole- 
cules, primary elicitors of damage, propagators, and signal molecules for the activation of defense or repair [43]. ROS can be detected histologically [44], however this approach is relatively laborious and destructive. Alternatively, ROS sensitive fluorescent probes allow real-time in vivo investigation of ROS production. Moreover, with an imaging approach ROS probes can quantify as well as visualize the temporal and spatial changes in the ROS production.

Numerous probes are available for specific or general ROS detection [41] [43] [45]-[50]. This approach was successfully used with higher plants [41] [42] [51] however, loading of the dye into the leaf material is problematic as the procedure can cause additional stress and thus additional ROS formation as well as the distribution of the dye is rather heterogeneous. To avoid this difficult to discriminate an uneven indicator distribution from an uneven ROS distribution, protoplasts are used instead of whole leaves which provides improved results [41]. Therefore, using a PA suspension cultures is an ideal compromise between the complex plant tissue and unicellular but fragile protoplasts as the probe is applied directly into the suspension of cells with intact cell walls.

\section{Potential of Fluorescence Imaging of PA Cell Suspension Cultures for Toxic Compound Screening}

As outlined above PA cell suspension cultures proved to be suitable higher plant experimental systems that combine the general advantages of cell suspension cultures to be able to determine even weak and transient physiological responses to external stimuli with the most important and prominent feature of higher plants, the carbon autotrophy due to the possession of chloroplast with photosynthetic activity [10]. The latter key metabolic process can be determined with high sensitivity and with both temporal and spatial resolution by PAM chlorophyll fluorescence imaging of photosystem activity [32]. Due to the specific nature of cell suspensions, they can be grown under standardized condition and uniformly distributed like microalgae in microtiter plates. Thus, PA cell suspension cultures are suitable as robust and simple higher plant high-throughput screening systems for toxicants where physiological responses such as the photosynthetic activity, secondary metabolite and ROS formation can be quantitatively and highly sensitively determined by fluorescence imaging techniques.

To obtain a proof of concept we have tested as a case study the impact of a photosynthesis inhibitor with known mode of action, 3-(3,4-dichloro-phenyl)1,1-dimethylurea (DCMU). The phytotoxicity of DCMU is caused by blocking electron transport flow in PSII at the QB site terminating the photosynthetic light reactions and causing formation of singlet oxygen which results in lipid peroxidation (reviewed in [52]). As plant material we have used PA cultures of the model plant tomato (Solanum peruvianum, [53]) that is also an important vegetable crop plant. In our case study, ten concentrations of DCMU and deionized water as a solvent control were pipetted into a 96-well microplate, in a volume of $100 \mu \mathrm{L}$ per well, four wells as replicates for each concentration. Thereafter, $100 \mu \mathrm{L}$ of cell suspension of a 14 days old PA suspension culture of $S$. peru- 
vianum was added. The DCMU concentrations were prepared to give final concentrations of $0.025 \mu \mathrm{M}$ to $40 \mu \mathrm{M}$ in the wells with PA culture cells. The microtiter plate was incubated in an illuminated growth incubator (AlgaeTron AG 230, PSI, CZ) under the usual cultivation conditions of $100 \mu$ mol photons $\mathrm{m}^{-2} \cdot \mathrm{s}^{-1}, 25^{\circ} \mathrm{C}$ and atmosphere enriched with $2 \% \mathrm{CO}_{2}$. Twenty minutes after the exposure of the cell suspension with the DCMU the cells were dark adapted for 10 minutes followed by the measurements of ChlF induction kinetics (Closed FluorCam FC 800-C, PSI, CZ). The imaging results are shown in Figure 1 and the quantitative evaluation of the data in Table 1.

Figure 1 and the quantitative evaluation thereof shown in Table 1 demonstrate that a concentration dependent negative effect of DCMU on photosynthetic performance, determined via the ChlF parameter Fv/Fm can be detected. Figure 1 illustrates the possibility to visualize and quantify a dose dependent response of PA cultures to DCMU treatment with ChlF imaging with high precision and low variability. Moreover, it is possible to detect the negative effect on the photosynthetic capacity at DCMU concentrations as low as $0.5 \mu \mathrm{M}$ DCMU within only 30 minutes after treatment.

The case study demonstrates that it is possible to detect in a physiological

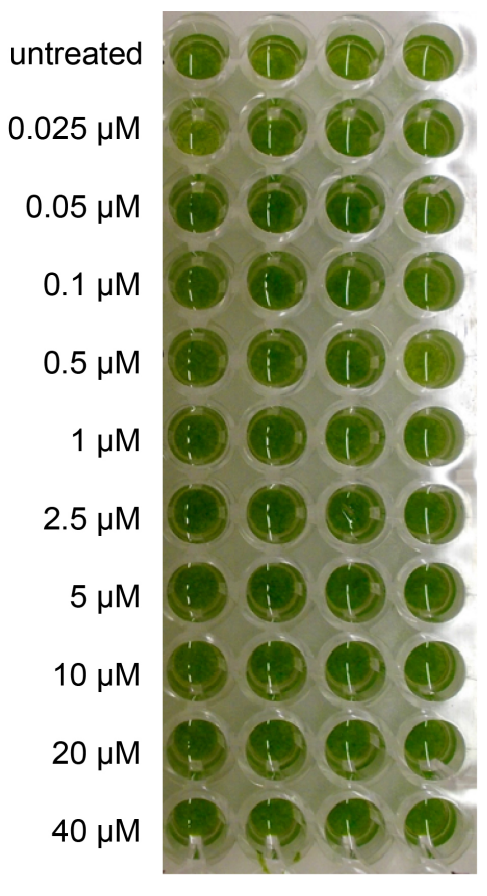

(a)

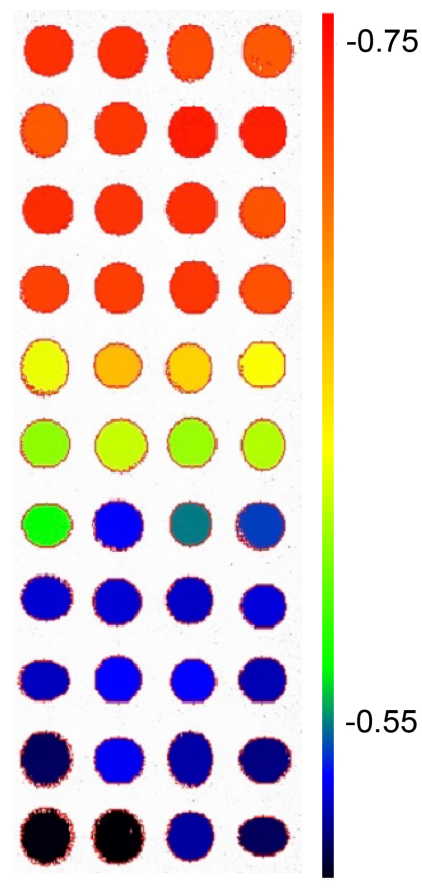

(b)

Figure 1. Response of photoautotrophic cell suspension of Solanum peruvianum in a 96-well microtiter plate 30 minutes after treatment with various concentrations of the photosynthesis inhibitor DCMU. (a) RGB image of the treated suspension; (b) Visualization of the PAM chlorophyll fluorescence imaging parameter Fv/Fm values as calculated by the FluorCam software and false color representation of the values. The scale shows the range of average values of the parameter normalized to the highest measured value. 
Table 1. Values of the PAM chlorophyll fluorescence imaging parameter Fv/Fm) of the Solanum peruvianum PA cell culture treated with DCMU for $30 \mathrm{~min}$ shown in Figure 1 (averages and SD from 4 repetitions).

\begin{tabular}{ccc}
\hline & \multicolumn{2}{c}{$\mathrm{Fv} / \mathrm{Fm}$} \\
\cline { 2 - 3 } Treatment & Average & $\mathrm{SD}$ \\
\hline $0.025 \mu \mathrm{M}$ & 0.73 & 0.01 \\
$0.05 \mu \mathrm{M}$ & 0.74 & 0.01 \\
$0.1 \mu \mathrm{M}$ & 0.74 & 0.01 \\
$0.5 \mu \mathrm{M}$ & 0.73 & 0.01 \\
$1 \mu \mathrm{M}$ & 0.65 & 0.02 \\
$2.5 \mu \mathrm{M}$ & 0.61 & 0.01 \\
$5 \mu \mathrm{M}$ & 0.55 & 0.02 \\
$10 \mu \mathrm{M}$ & 0.53 & 0.00 \\
$20 \mu \mathrm{M}$ & 0.53 & 0.01 \\
$40 \mu \mathrm{M}$ & 0.52 & 0.01 \\
& 0.51 & 0.01 \\
\hline
\end{tabular}

phenotyping approach [54] the response of photosynthesis, as the key physiological pathway of higher plants, in PA cell cultures at low toxicants concentrations as well as short times and with low variability. It is apparent that the physiological responses can be detected long before a negative impact is phenotypically visible (Figure 1(a)) and thus pre-symptomatically. In previous studies, it has been shown that the response of PA cultures is typically faster and more sensitive compared to the reactions of organs in whole plants. Definitely this approach is much faster than the current standard toxicant tests with higher plants that measure growth responses and thus the required time is largely reduced from weeks to only minutes or hours. Due to the test in microtiter plate with at least 96 wells the throughput capacity is much higher compared to plants grown individually in pots in greenhouses and therefore the demand for required infrastructures, space, consumables and personnel is very much reduced. As outlined above, in addition to photosynthesis also other established parameters indicative of plant stress responses can be sensitively and quantitatively measured by fluorescence imaging. Since important key physiological responses are measured it is also possible to elucidate, eventually in combination with additional complementary physiological, biochemical and molecular methods, the mode of action of the tested toxicants. There is already a long experience in the use of the suggested fluorescence detection methods in basic and applied plant science stress response research. In summary, the combination of photoautotrophic cultures with the determination of fluorescence signatures presents a promising experimental approach for establishing a highly sensitive, rapid high-throughput screening system of plant sensitivity to toxic compounds.

Although toxicity screening by plant cell cultures had been principally considered before, the various serious practical limitation discussed for their practical use [55] are only overcome by the specific combination of PA cultures with flu- 
orescence imaging techniques outlined above. The potential for screening of herbicides with plant cell cultures and imaging technology was already proposed before [16], however, so far image analysis was only used for counting callus colonies as a measure of growth inhibition. We rather propose to use autotrophic cultures, measure different biomarkers and use image analysis for high-throughput modification of standard stress physiology tests. The proposed experimental test system involves both general physiological reactions, such as the inhibition of photosynthesis, as well as specific cellular responses, such as the formation of specific secondary metabolites, so additional information on toxicology and physiology compared to the standardly measured growth inhibition in ecotoxicology can be obtained. Finally, the test system can be integrated in functional, physiological phenotyping analyses and it is possible, in combination with MCF and ROS detection, to even derive information on the particular subcellular distribution of the responses.

Despite the various advantages of establishing toxicity tests based on the fast and sensitive determination of responses of PA suspension in microtiter plates by fluorescence imaging, this novel system has also certain limitations. In cases where a specific uptake mechanism of a toxicant in a particular plant organ is relevant, e.g. the uptake from the soil via the root system, the results obtained from PA cultures are possibility misleading in terms of overestimating a possible impact [16]. Likewise, if a toxicity is dependent on the occurrence of specific metabolic conversions of a compound which are not possible in the cell cultures a toxicity may be underestimated [56]. This also applies if a particular cellular target is not expressed in the dedifferentiated cells. Finally, a particular toxic compound may react, become degraded or inactivated in combination with the particular culture medium [18]. Therefore, the PA culture assay will not make the classic plant growth assays completely obsolete but complement them in particular for large scale prescreening and for elucidation of the mode of action.

\section{Conclusion and Observations}

Despite the increasing need to assess the impact of known as well as new toxicants on higher plants in general and environmentally relevant toxicants on crop plants in particular, so far there is a lack of sensitive, physiologically relevant, high-throughput and fast higher plant test systems. As outlined above, although being used in basic research for more than 40 years, higher plant photoautotrophic suspension culture cells are so far an unexplored experimental platform in toxicology that combines the advantage of the ease of handling microalgae for high-throughput screening and characterization in microtiter plates with the possibility to assess physiological responses of primary and secondary metabolism of higher plants fast, highly sensitive and with high precision by fluorescence imaging. The successful application of PA suspension cultures in different topics of basic science research has demonstrated that physiological responses and even complex coordinated regulatory mechanisms of PA suspension cultures correspond to those of intact higher plants and in some cases even pro- 
vided the basis to identify such mechanisms in higher plant-microbe interactions. The physiological responses of PA cultures are thus verified proxy for responses in plant organs, most importantly in the biggest and for crop plant production most relevant above ground organ, the photosynthetically active leaves. The in vitro grown PA cultures ensure a supply of cells of constant quality and make the need for special greenhouse infrastructures and personal resources obsolete as well as the medium and long term planning to have plants at a certain developmental stage available that is not influenced by changing environmental conditions throughout the year. Therefore, we suggest considering supported by the results of a case study shown above, the use of the various available PA suspension cultures of well-established model and agronomically relevant crop plants as valuable experimental platform for toxicology research. Thus, the great potential of the PA cultures as experimental ecotoxicology test system should be systematically explored and verified. Although it is not expected that PA culture will be able to substitute intact plants completely, the PA cultures could function as a first and fast screening system that allows a much higher throughput with an increased sensitivity and robustness. An additional advantage of PA cultures is the easier possibility to elucidate the mode of action of a particular toxicant especially when combined with proposed imaging techniques of chlorophyll fluorescence, multicolor fluorescence and ROS fluorescent probes. The ultimate goal needs to be the validation and comparison of newly established PA test systems with e.g. standard OECD test systems to ultimately complement and extend the range of available, standardized test systems. The most efficient way for exploring and validating the apparent potential of PA cultures as toxicant screening and test system would be a holistic, interdisciplinary approach and could be most efficiently accomplished by the interdisciplinary collaboration between plant physiologists and toxicology research institutions for the sake of environmental safety and healthy food production.

\section{Acknowledgements}

The presentation of this research concept as a poster at the INEF 2016 conference was supported by the International Network of Environmental Forensics. Anna Segečvá, Jan Cervený and Thomas Roitsch were supported by the Ministry of Education, Youth and Sports of CR within the National Sustainability Program I (NPU I), grant number LO1415. Jan Červený was also supported by GA CR, grant number 15-17367S. Access to instruments and other facilities was supported by the Czech research infrastructure for systems biology C4SYS (project no LM2015055). The authors would like to thank PSI-Photon Systems Instruments, spol. s r.o. (Drasov, Czech Republic) for providing access to Closed FluorCam FC 800-C and in particular Dr. Klára Panzarová for helpful discussion and technical advice and support.

\section{References}

[1] OECD (2006) Test No. 208: OECD Guidelines for the Testing of Chemicals. Terre- 
strial Plant Test: Seedling Emergence and Seedling Growth Test. Organisation for Economic Co-Operation and Development, Paris.

[2] OECD (2006) Test No. 227: OECD Guidelines for Testing of Chemicals. Terrestrial Plant Test: Vegetative Vigour Test. Organisation for Economic Co-Operation and Development, Paris.

[3] OECD (2006) Test No. 221: Lemna sp. Growth Inhibition Test. Organisation for Economic Co-Operation and Development, Paris.

[4] OECD (2011) Test No. 201: OECD Guidelines for the Testing of Chemicals. Freshwater Alga and Cyanobacteria, Growth Inhibition Test. Organisation for Economic Co-operation and Development, Paris.

[5] Klaine, S., Lewis, M. and Knuteson, S. (2003) Phytotoxicity. In: Hoffman, D., Rattner, B. and Burton, G., Eds., Handbook of Ecotoxicology, Lewis Publishers, 191218.

[6] Grimme, L.H., Riess, M.H., Manthey, M., Faust, M. and Altenburger, R. (1993) Cell Physiological Parameters to Detect Ecotoxicological Risks. Science of the Total Environment, 134, 741-748.

[7] Inderjit, Saini, M. and Kaur, H. (2005) Experimental Complexities in Evaluating the Comparative Phytotoxicity of Chemicals with Different Modes of Action. Environmental and Experimental Botany, 53, 97-104.

[8] Kawabata, Y. and Takeda, S. (2014) Regulation of Xanthophyll Cycle Pool Size in Response to High Light Irradiance in Arabidopsis. Plant Biotechnology, 31, 229 240. https://doi.org/10.5511/plantbiotechnology.14.0609a

[9] Sato, F. (2013) Characterization of Plant Functions Using Cultured Plant Cells, and Biotechnological Applications. Bioscience, Biotechnology, and Biochemistry, 77, 19. https://doi.org/10.1271/bbb.120759

[10] Roitsch, T. and Sinha, A. (2002) Application of Photoautotrophic Suspension Cultures in Plant Science. Photosynthetica, 40, 481-492. https://doi.org/10.1023/A:1024332430494

[11] Gómez-Torres, L.M., Moreno-Gómez, B., Velásquez-Lozano, M.E., Aguirre-Mancilla, C. and Aguado-Santacruz, G.A. (2014) Plant Cell Photoautotrophic Suspension Cultures. Establishment and Application Perspectives. Revista Fitotecnia Mexicana, 37, 165-179.

[12] Doran, P.M. (2009) Application of Plant Tissue Cultures in Phytoremediation Research: Incentives and Limitations. Biotechnology and Bioengineering, 103, 60-76. https://doi.org/10.1002/bit.22280

[13] Santos, R.B., Abranches, R., Fischer, R., Sack, M. and Holland, T. (2016) Putting the Spotlight Back on Plant Suspension Cultures. Frontiers in Plant Science, 7, 1-13. https://doi.org/10.3389/fpls.2016.00297

[14] Harms, H.H. (1992) In Vitro Systems for Studying Phytotoxicity and Metabolic Fate of Pesticides and Xenobiotics in Plants. Pesticide Science, 35, 277-281. https://doi.org/10.1002/ps.2780350313

[15] Schwenger-Enger, C., Barz, W. and Weber, N. (2001) Fatty Acid Alteration of Plastidic and Extra-Plastidic Membrane Lipids in Metribuzin-Resistant Photoautotrophic Chenopodium rubrum Cells as Compared to Wild-Type Cells. Verlag Der Zeitschrift Für Naturforschung, 56, 1047-1056.

[16] Olofsdotter, M., Olesen, A., Andersen, S.B. and Streibig, J.C. (1994) A Comparison of Herbicide Bioassays in Cell Cultures and Whole Plants. Weed Research, 34, 387 394. https://doi.org/10.1111/j.1365-3180.1994.tb02034.x

[17] Thiemann, J., Nieswandt, A. and Barz, W. (1989) A Microtest System for the Serial 
Assay of Phytotoxic Compounds Using Photoautotrophic Cell Suspension Cultures of Chenopodium rubrum. Plant Cell Reports, 8, 399-402. https://doi.org/10.1007/BF00270078

[18] Zilkah, S. and Gressel, J. (1977) Cell Cultures vs. Whole Plants for Measuring Phytotoxicity I. The Establishment and Growth of Callus and Suspension Cultures; Definition of Factors Affecting Toxicity on Calli. Plant and Cell Physiology, 18, 641655.

[19] Roitsch, T. and Sinha, A. (2001) Effect of Different Sugars on Photosynthesis and Chlorophyll Fluorescence in Photoautotrophic Tomato Suspension Cell Cultures. Photosynthetica, 39, 611-614. https://doi.org/10.1023/A:1015624600607

[20] Hüsemann, W. (1985) Photoautotrophic Growth of Cells in Culture. In: Vasil, I.K., Ed., Cell Culture and Somatic Cell Genetics of Plants, Vol. 2, Academic Press, New York, 213-251.

[21] Widholm, J.M. (1992) Properties and Uses of Photoautotrophic Plant Cell Cultures. International Review of Cytology, 132, 109-175.

[22] Hofmann, M., Ehneß, R., Lee, T.K. and Roitsch, T. (1999) Intracellular Protons Are Not Involved in Elicitor Dependent Regulation of mRNAs for Defense Related Enzymes in Chenopodium rubrum. Journal of Plant Physiology, 155, 527-532.

[23] Sano, T., Higaki, T., Handa, K., Kadota, Y., Kuchitsu, K., Hasezawa, S., Hoffmann, A., Zimmermann, U., Hedrich, R. and Roitsch, T. (2006) Calcium Ions Are Involved in the Delay of Plant Cell Cycle Progression by Abiotic Stresses. FEBS Letters, 580, 597-602.

[24] Ehness, R., Ecker, M., Godt, D.E. and Roitsch, T. (1997) Glucose and Stress Independently Regulate Source and Sink Metabolism and Defense Mechanisms via Signal Transduction Pathways Involving Protein Phosphorylation. The Plant Cell, 9, 1825-1841. https://doi.org/10.1105/tpc.9.10.1825

[25] Berger, S., Sinha, A.K. and Roitsch, T. (2007) Plant Physiology Meets Phytopathology: Plant Primary Metabolism and Plant-Pathogen Interactions. Journal of Experimental Botany, 58, 4019-4026. https://doi.org/10.1093/jxb/erm298

[26] Fai, P.B., Grant, A. and Reid, B. (2007) Chlorophyll a Fluorescence as a Biomarker for Rapid Toxicity Assessment. Environmental Toxicology and ChemistrylSETAC, 26, 1520-1531. https://doi.org/10.1897/06-394R1.1

[27] Chaerle, L., Lenk, S., Leinonen, I., Jones, L., Van Der Straeten, D. and Buschmann, C. (2009) Multi-Sensor Imaging of Plant Stresses: Towards the Development of a Stress-Catalogue. Biotechnology Journal, 4, 1152-1167. https://doi.org/10.1002/biot.200800242

[28] Baker, N.R. (2008) Chlorophyll Fluorescence: A Probe of Photosynthesis in Vivo. Annual Review of Plant Biology, 59, 89-113. https://doi.org/10.1146/annurev.arplant.59.032607.092759

[29] Maxwell, K. and Johnson, G.N. (2000) Chlorophyll Fluorescence-A Practical Guide. Journal of Experimental Botany, 51, 659-668.

[30] Lichtenthaler, H.K., Langsdorf, G., Lenk, S. and Buschmann, C. (2005) Chlorophyll Fluorescence Imaging of Photosynthetic Activity with the Flash-Lamp Fluorescence Imaging System. Photosynthetica, 43, 355-369. https://doi.org/10.1007/s11099-005-0060-8

[31] Barbagallo, R., Oxborough, K., Pallett, K. and Baker, N. (2003) Rapid, Noninvasive Screening for Perturbations of Metabolism and Plant Growth Using Chlorophyll Fluorescence Imaging. Plant Physiology, 132, 485-493.

https://doi.org/10.1104/pp.102.018093 
[32] Nedbal, L., Soukupová, J., Kaftan, D., Whitmarsh, J. and Trtílek, M. (2000) Kinetic Imaging of Chlorophyll Fluorescence Using Modulated Light. Photosynthesis Research, 66, 3-12. https://doi.org/10.1023/A:1010729821876

[33] Harbinson, J., Prinzenberg, A.E., Kruijer, W. and Aarts, M.G.M. (2012) High Throughput Screening with Chlorophyll Fluorescence Imaging and Its Use in Crop Improvement. Current Opinion in Biotechnology, 23, 221-226.

[34] Baker, N.R. and Rosenqvist, E. (2004) Applications of Chlorophyll Fluorescence Can Improve Crop Production Strategies: An Examination of Future Possibilities. Journal of Experimental Botany, 55, 1607-1621. https://doi.org/10.1093/jxb/erh196

[35] Gorbe, E. and Calatayud, A. (2012) Applications of Chlorophyll Fluorescence Imaging Technique in Horticultural Research: A Review. Scientia Horticulturae, 138, 24-35.

[36] Wilkinson, A.D., Collier, C.J., Flores, F., Mercurio, P., O’Brien, J., Ralph, P.J. and Negri, A.P. (2015) A Miniature Bioassay for Testing the Acute Phytotoxicity of Photosystem II Herbicides on Seagrass. PLoS ONE, 10, e0117541. https://doi.org/10.1371/journal.pone.0117541

[37] Lichtenthaler, H.K., Lang, M., Sowinska, M., Heisel, F. and Miehé, J.A. (1996) Detection of Vegetation Stress via a New High Resolution Fluorescence Imaging System. Journal of Plant Physiology, 148, 599-612.

[38] Buschmann, C. and Lichtenthaler, H.K. (1998) Principles and Characteristics of Multi-Colour Fluorescence Imaging of Plants. Journal of Plant Physiology, 152, 297-314.

[39] Morales, F., Cerovic, Z.G. and Moya, I. (1994) Characterization of Blue-Green Fluorescence in the Mesophyll of Sugar Beet (Beta vulgaris L.) Leaves Affected by Iron Deficiency. Plant Physiology, 106, 127-133. https://doi.org/10.1104/pp.106.1.127

[40] Karlický, V., Nezval, J., Štroch, M. and Špunda, V. (2013) Response of Epidermal Blue-Green Fluorescence Emission from Barley Leaves to UV Radiation Stress. In: Stojanov, R., Ed., Global Change and Resilience: From Impacts to Responses, Global Change Research Centre, Academy of Sciences of the Czech Republic, Brno, 231-235.

[41] Kristiansen, K.A., Jensen, P.E., Møller, I.M. and Schulz, A. (2009) Monitoring Reactive Oxygen Species Formation and Localisation in Living Cells by Use of the Fluorescent Probe CM-H2DCFDA and Confocal Laser Microscopy. Physiologia Plantarum, 136, 369-383. https://doi.org/10.1111/j.1399-3054.2009.01243.x

[42] Swanson, S.J., Choi, W., Chanoca, A. and Gilroy, S. (2011) In Vivo Imaging of $\mathrm{Ca} 2+, \mathrm{pH}$, and Reactive Oxygen Species Using Fluorescent Probes in Plants. Annual Review of Plant Biology, 62, 273-297. https://doi.org/10.1146/annurev-arplant-042110-103832

[43] Hideg, E., Barta, C., Kálai, T., Vass, I., Hideg, K. and Asada, K. (2002) Detection of Singlet Oxygen and Superoxide with Fluorescent Sensors in Leaves under Stress by Photoinhibition or UV Radiation. Plant \& Cell Physiology, 43, 1154-1164. https://doi.org/10.1093/pcp/pcf145

[44] Jambunathan, N. (2010) Determination and Detection of Reactive Oxygen Species (ROS), Lipid Peroxidation, and Electrolyte Leakage in Plants. In: Sunkar, R., Ed., Plant Stress Tolerance: Methods and Protocols, Springer, Berlin, 291-297. https://doi.org/10.1007/978-1-60761-702-0_18

[45] Hideg, E., Kálai, T., Kós, P.B., Asada, K. and Hideg, K. (2006) Singlet Oxygen in Plants-Its Significance and Possible Detection with Double (Fluorescent and Spin) Indicator Reagents. Photochemistry and Photobiology, 82, 1211-1218. https://doi.org/10.1562/2006-02-06-RA-797 
[46] Hideg, É. (2008) A Comparative Study of Fluorescent Singlet Oxygen Probes in Plant Leaves. Central European Journal of Biology, 3, 273-284. https://doi.org/10.2478/s11535-008-0018-5

[47] Kálai, T., Hideg, É., Ayaydin, F. and Hideg, K. (2013) Synthesis and Potential Use of 1,8-Naphthalimide Type (1) O2 Sensor Molecules. Photochemical \& Photobiological Sciences: Official Journal of the European Photochemistry Association and the European Society for Photobiology, 12, 432-438.

https://doi.org/10.1039/C2PP25253H

[48] Owusu-Ansah, E., Yavari, A. and Banerjee, U. (2008) A Protocol for in Vivo Detection of Reactive Oxygen Species. Protocol Exchange, Nature Publishing Group.

[49] Šnyrychová, I., Ayaydin, F. and Hideg, É. (2009) Detecting Hydrogen Peroxide in Leaves in Vivo-A Comparison of Methods. Physiologia Plantarum, 135, 1-18. https://doi.org/10.1111/j.1399-3054.2008.01176.x

[50] Srivastava, R.K., Pandey, P., Rajpoot, R., Rani, A. and Dubey, R.S. (2014) Cadmium and Lead Interactive Effects on Oxidative Stress and Antioxidative Responses in Rice Seedlings. Protoplasma, 251, 1047-1065.

https://doi.org/10.1007/s00709-014-0614-3

[51] Hideg, É. and Schreiber, U. (2007) Parallel Assessment of ROS Formation and Photosynthesis in Leaves by Fluorescence Imaging. Photosynthesis Research, 92, 103 108. https://doi.org/10.1007/s11120-007-9146-4

[52] Dewez, D., Marchand, M., Eullaffroy, P. and Popovic, R. (2002) Evaluation of the Effects of Diuron and Its Derivatives on Lemna Gibba Using a Fluorescence Toxicity Index. Environmental Toxicology, 17, 493-501. https://doi.org/10.1002/tox.10084

[53] Link, V.L., Hofmann, M.G., Sinha, A.K., Ehness, R., Strnad, M. and Roitsch, T. (2002) Biochemical Evidence for the Activation of Distinct Subsets of Mitogen-Activated Protein Kinases by Voltage and Defense-Related Stimuli 1. Plant Physiology, 128, 271-281. https://doi.org/10.1104/pp.010569

[54] Großkinsky, D.K., Svensgaard, J., Christensen, S. and Roitsch, T. (2015) Plant Phenomics and the Need for Physiological Phenotyping across Scales to Narrow the Genotype-to-Phenotype Knowledge Gap. Journal of Experimental Botany, 66, 5429 5440. https://doi.org/10.1093/jxb/erv345

[55] Kristen, U. (1997) Use of Higher Plants as Screens for Toxicity Assessment. Toxicology in Vitro, 11, 181-191.

[56] Edwards, R. and Owen, W.J. (1986) Comparison of Glutathione S-Transferases of Zea mays Responsible for Herbicide Detoxification in Plants and Suspension-Cultured Cells. Planta, 169, 208-215. https://doi.org/10.1007/BF00392316 


\section{Abbreviations}

CCD, charge coupled device

ChlF, chlorophyll fluorescence

DCMU, 3-(3,4-dichlorophenyl)-1,1-dimethylurea

Fv/Fm, maximum quantum efficiency of PSII photochemistry

MCF, multicolor fluorescence

PA, photoautotrophic

PAM fluorescence, pulse amplitude modulated fluorescence

PSII, photosystem II

$\mathrm{QB}$, secondary quinone electron acceptor of PSII

ROS, reactive oxygen species

Submit or recommend next manuscript to SCIRP and we will provide best service for you:

Accepting pre-submission inquiries through Email, Facebook, LinkedIn, Twitter, etc. A wide selection of journals (inclusive of 9 subjects, more than 200 journals)

Providing 24-hour high-quality service

User-friendly online submission system

Fair and swift peer-review system

Efficient typesetting and proofreading procedure

Display of the result of downloads and visits, as well as the number of cited articles

Maximum dissemination of your research work

Submit your manuscript at: http://papersubmission.scirp.org/

Or contact jep@scirp.org 